\title{
A Medical Image Steganography Method based on Integer Wavelet Transform and Overlapping Edge Detection
}

\author{
Hayat Al-Dmour and Ahmed Al-Ani \\ Faculty of Engineering and Information Technology, University of Technology, \\ Sydney, Ultimo NSW 2007 Australia \\ Hayat.Dmour@uts.edu.au, Ahmed.Al-Ani@uts.edu.au
}

\begin{abstract}
Recently, there has been an increased interest in the transmission of digital medical images for e-health services. However, existing implementations of this service do not pay much attention to the confidentiality and protection of patients' information. In this paper, we present a new medical image steganography technique for protecting patients' confidential information through the embedding of this information in the image itself while maintaining high quality of the image as well as high embedding capacity. This technique divides the cover image into two areas, the Region of Interest (ROI) and the Region of Non-Interest (RONI), by performing Otsu's method and then encloses ROI pixels in a rectangular shape according to the binary pixel intensities. In order to improve the security, the Electronic Patient Records (EPR) is embedded in the high frequency sub-bands of the wavelet transform domain of the RONI pixels. An edge detection method is proposed using overlapping blocks to identify and classify the edge regions. Then, it embeds two secret bits into three coefficient bits by performing an XOR operation to minimize the difference between the cover and stego images. The experimental results indicate that the proposed method provides a good compromise between security, embedding capacity and visual quality of the stego images.
\end{abstract}

Keywords - Information hiding, Steganography, edge detection, human visual system (HVS), Wavelet Transform, ROI, and RONI.

\section{Introduction}

Medical Information Systems (MIS) and Information Technology (IT) form the infrastructure of Telemedicine that incorporates computer to store, process and exchange medical information remotely [1]. During transmission, digital medical images and confidential patients' information should be protected from attackers. Therefore, different techniques have been presented to provide protection of medical images and electronic patient records (EPR) [2]. These methods are based either on cryptography or steganography techniques. Cryptography methods use encoding to hide the meaning of the message, but they cannot hide its existence [3]. However, the transmission of encoded text certainly stimulate intruders' attention, whom may try to decode it. Steganography on the other hand 
is the art of concealing the existence of the secret message within other media, such as text, image, audio and video without drawing attention of unintended users $[2,4]$. Combining steganography and cryptography aim to make it difficult for attackers to extract the sensitive information [3]. This type of integration does not embed readable text, but embeds encrypted text to add another layer of security.

The Least Significant Bit (LSB) method is the most common steganography methodology $[2,5]$. It is capable of embedding large amount of secret data in a cover image, however it is vulnerable to statistical attacks [6].

In this paper, we propose a new medical image steganography for hiding the EPR into the coefficients of the Integer Wavelet Transform (IWT) high sub-bands to achieve high level of imperceptibility and security. The embedding process is accomplished in eight steps: (i) separate the cover image into two regions, ROI and RONI, (ii) reconstruct RONI pixels into a square matrix, (iii) histogram modification is performed on RONI matrix, (iv) the modified RONI is transformed using 2D IWT to get four sub-bands, (v) implement the detection of edge locations in high frequency sub-bands, (vi) apply an XOR operation, which minimizes the degradation caused by embedding the secret message, (vii) apply inverse IWT to recover RONI pixels, and (viii) combine the RONI to

get the stego image. Our proposed algorithm utilizes the visual characteristic of overlapping blocks of pixels such that it achieves better capacity and maintains high visual quality.

The rest of this paper is organized as follows. The related work is described in section 2. Details of the proposed method are presented in section 3. Section 4 presents the experimental results, and the conclusion is given in section 5 .

\section{Related Work}

Exchange of medical information between hospitals and medical clinics placed in different areas is a habitual practice. Therefore, numerous methods have been introduced to provide secure storage and transmission using information hiding techniques.

Navas et. al. [7] introduced a blind and reversible data hiding scheme for telemedicine applications that depends on IWT. The ROI is manually identified in the low coefficients sub-band as a rectangle shape. In order to obviate misdiagnosis, encrypted EPR is embedded in RONI and the ROI is stored without any noise. However, this method can hide at most 3400 characters into a cover image of size $512 \times 512$ and the computational cost is high. Zou et al. [8] proposed a reversible watermarking method to embed the secret data into the IWT high frequency sub-bands. The embedding procedure utilizes the high frequency sub-bands, where it divides each sub-band into non-overlapping blocks and performs a shifting operation to embed the data. This method is robust to JPEG2000 compression, however, it achieves low embedding rate. Prabakaran et. al. proposed a method that utilizes IWT to secure an MRI medical image into a single holder. The method treats the patient's medical diagnosis image as a mystery image and applies the Arnold transform before a acquiring a scrambled 
secret image, which is hidden into a dummy holder, and the Inverse IWT is applied to get the secret image [9]. In [10], Solanki et. al. proposed a watermarking scheme to hide the encrypted watermark in the CT or MRI images to ensure the security of EPR. This method begins by separating the original image into ROI and RONI. It identifies ROI by subtracting the cover image from the negative image, where the negative image is computed by subtracting the medical image from 255. To improve the security, the watermark is encoded by applying the RSA encryption method. Blockwise DWT is then performed over the image.

To overcome some of the drawbacks of the existing methods (i.e., the low embedding rates, low visual quality, underflow/overflow problem and protect ROI from any modifications), this paper proposes a new information hiding method in the IWT domain to embed the secret data in the edge regions of the high frequency sub-bands.

\section{The Proposed Method}

\subsection{The Embedding Process}

Almost every medical image contains a particularly important area that is known as the Region of Interest (ROI). This region should not be altered, and hence, when a steganography algorithm is applied the embedding of EPR data should be carried out in the Region of Non-Interest (RONI) only. The implementation of the embedding process is illustrated in Figure 1 and explained in the following steps.

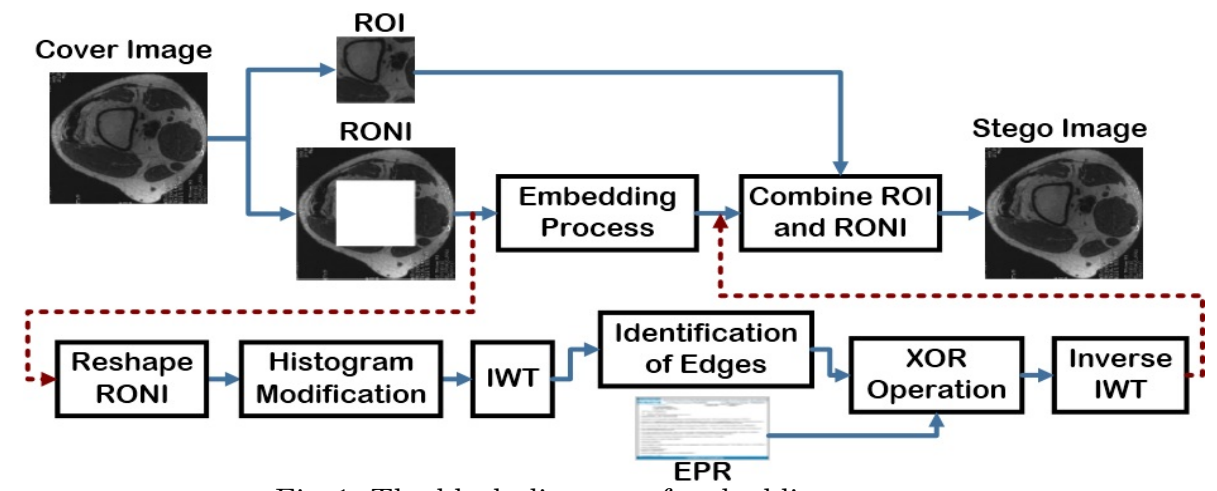

Fig. 1: The block diagram of embedding process.

Algorithm 1: The Embedding Process.

Inputs: Cover image $(C)$, Secret data $(M)$, block size $(n \times n$, which is expected here to be $4 \times 4)$, threshold value $(T h)$.

Output: Stego image $(S)$.

1. Identification of ROI and RONI: The Otsu's method is utilized to identify the threshold value that reduces the difference of the black and white pixels. To separate the cover image into two regions, a threshold value is defined to differentiate between ROI and RONI i.e., the pixel is related to ROI, if 


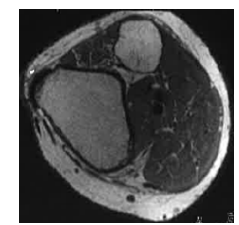

(a)

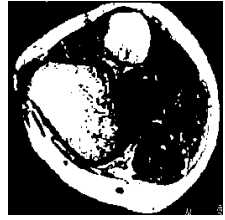

(b)

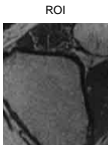

(c)

Fig. 2: (a) Cover Image $256 \times 256$, (b) Binary image , and (C) ROI.

the pixel value is greater than the threshold value. Otherwise, it is related to RONI. Figures 2(a) and 2(b) show the cover and binary images resulting from the Otsu's method. The ROI is selected in rectangular shape according to the white pixel intensity, as shown in Figure 2(c).

2. Reshape RONI: RONI pixels are reshaped into a square matrix of size $r \times r$.

3. Preprocessing: The underflow/overflow problem occurs with the existence of pixels whose grey level values are near 0 or 255 . This issue can be rectified by enforcing upper and lower limits (240 and 15 respectively) to the pixel's grey level value.

4. Integer Wavelet Transform (IWT): Apply the first level of IWT on the modified square of RONI to decompose the RONI into four sub-bands (LL1, HL1, LH1 and HH1). This will be followed by concatenating the HH1, LH1 and HL1 sub-bands in vertical order. In order to improve the embedding capacity and to detect the edge regions accurately, the absolute value is applied to the high sub-bands to generate positive coefficients. Also, the original coefficient sign $\left(T_{\text {sign }}\right)$ is preserved in a temporary matrix to ensure a good quality of the stego image.

5. Identification of Edges: According to the fact that human eyes can bear more modifications in edge regions compared to uniform regions, we attempt to improve the stego image quality through embedding the message bits into edge regions. This will be achieved through an efficient edge detection scheme that uses overlapping blocks to identify and classify image blocks according to their edge strength, where more bits can be hidden into the strong edges and less bits into the smooth ones. Moreover, the aim of using overlapping blocks is to enhance the embedding capacity by reducing the number of unused pixels. The implementation of this process is explained in the following steps:

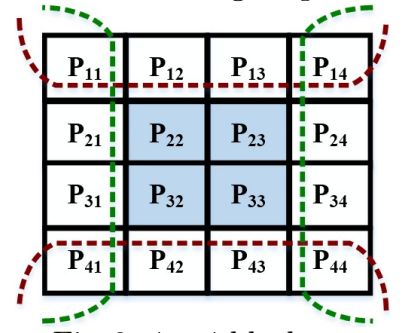

$$
\begin{gathered}
1^{\text {st }} D=\operatorname{avg}\left|\left[P_{12}, P_{13}, P_{14}, P_{24}, P_{34}\right]-\left[P_{21}, P_{31}, P_{41}, P_{42}, P_{43}\right]\right| \\
2^{\text {nd }} D=\operatorname{avg}\left|\left[P_{13}, P_{12}, P_{11}, P_{21}, P_{31}\right]-\left[P_{24}, P_{34}, P_{44}, P_{43}, P_{42}\right]\right| \\
H \quad=\operatorname{avg}\left|\left[P_{11}, P_{12}, P_{13}, P_{14}\right]-\left[P_{41}, P_{42}, P_{43}, P_{44}\right]\right| \\
\quad=\operatorname{avg}\left|\left[P_{11}, P_{21}, P_{31}, P_{41}\right]-\left[P_{14}, P_{24}, P_{34}, P_{44}\right]\right|
\end{gathered}
$$

Fig. 3: $4 \times 4$ block

(a) Divide the image into overlapping blocks of size $4 \times 4$, as shown in Figure 3 , i.e., shifting of three columns/rows. 
(b) Image edges are classified into four directions. Therefore for each block, the magnitude value for each direction is computed using Eq. 1.

(c) The final magnitude value $(e)$ of each block is the maximum of the four values. The secret data $(M)$ is embedded into the four shaded pixels $\left(P_{22}, P_{23}, P_{32}\right.$, and $\left.P_{33}\right)$.

To minimize the computational cost, edge detection method is applied only once using a threshold of value 4 . Then, edge blocks are classified into five groups according to the magnitude value $(e)$ as shown in Table 1, where sharper edges, such as $G_{5}$ and $G_{4}$, have a higher priority than the weaker edges, such as $G_{1}$ and $G_{2}$.

Table 1: Numbers of bits that can be embedded in each of coefficients of an edge block based on the group it belongs to.

\begin{tabular}{|l|c|c|c|c|c|}
\hline Group & G1 & G2 & G3 & G4 & G5 \\
\hline Range of Group & {$[4-7]$} & {$[8-15]$} & {$[16-63]$} & {$[64-127]$} & {$[128-255]$} \\
\hline n (bpp) & 1 bit & 2 bits & 3 bits & 3 bits & 3 bits \\
\hline
\end{tabular}

6. The XOR operation: n-bits are embedded in each RONI edge coefficient, where the value of $n$ is determined by the magnitude value $(e)$ of each block. The algorithm begins with $G_{5}$ coefficients by utilizing three bits from each coefficient to embed the secret message. If $G_{5}$ coefficients are not enough for embedding, then $G_{4}$ coefficients are utilized (three bits from each coefficient). If the message is not fully embedded, then move to the lower priority groups ( $G_{3}$ then $G_{2}$ and finally $G_{1}$ ). The XOR operations are applied to embed two secret bits $m_{1}$ and $m_{2}$ into three bits $p_{1}, p_{2}$, and $p_{3}$ of the RONI coefficients as follows: $k_{1}=p_{1} \oplus p_{2}, k_{2}=p_{2} \oplus p_{3}$.

To complete the embedding process, the two secert bits $m_{1}$ and $m_{2}$ are compared to the calculated bits $k_{1}$ and $k_{2}$. There are four cases for this comparison:

$$
\begin{aligned}
& m_{1}=k_{1} \text { and } m_{2}=k_{2} \Rightarrow \text { no change } \\
& m_{1} \neq k_{1} \text { and } m_{2}=k_{2} \Rightarrow p_{1}=p_{1} \prime \\
& m_{1}=k_{1} \text { and } m_{2} \neq k_{2} \Rightarrow p_{2}=p_{2} \prime \\
& m_{1} \neq k_{1} \text { and } m_{2} \neq k_{2} \Rightarrow p_{3}=p_{3} \prime
\end{aligned}
$$

Equation 2 shows that embedding two secret bits into three coefficients bits requires the modification of at most one bit. When the embedding process is complete, the original coefficient sign $\left(T_{\text {sign }}\right)$ is assigned to the high frequency sub-bands.

7. Inverse IWT: Apply Inverse IWT on the four sub-bands to recover the RONI image of size $r \times r$.

8. Combine the ROI and RONI after the embedding process to construct the stego image. 


\subsection{The Extraction Process}

Extraction of the secret message bits is implemented as follows:

1. Divide the stego image into ROI and RONI by applying the Otsu method.

2. RONI pixels are reshaped into a square matrix of size $r \times r$.

3. Apply the first level of IWT transform on RONI from the stego image to decompose the RONI into four sub-bands (LL1, HL1, LH1 and HH1). Concatenate HH1, LH1 and HL1 in vertical order and find the absolute value of the resulting matrix.

4. Identification of Edges: Identify the edge regions in the high frequency subbands by performing the edge detection procedure used in the embedding process. Then, classify the edge blocks into five groups. Finally, the secret bits $m_{1}$ and $m_{2}$ are extracted from the three stego bits $q_{1}, q_{2}$, and $q_{3}$ using the following XOR extraction operations as follows: $m_{1}=q_{1} \oplus q_{2}, m_{2}=q_{2} \oplus q_{3}$.

\section{Experimental Result and Discussion}

The performance of the proposed method has been tested using 100 MRI images of size $256 \times 256$. The visual quality of the stego images are measured using the Peak Signal-to-Noise Ratio $(P S N R)$ which is defined in Eq. 3.

$$
P S N R=10 \log _{10}\left[\frac{255^{2} \times W \times H}{\sum_{i=1}^{W} \sum_{j=1}^{H}\left(c_{i j}-s_{i j}\right)^{2}}\right](d B)
$$

where $W$ and $H$ are the width and height of the cover image respectively, and $c_{i j}$ and $s_{i j}$ are the gray values of pixel $(i, j)$ of the cover and stego images respectively.

The weighted Peak Signal-to-Noise Ratio $(w P S N R)$ is an alternative image quality measurement. It improves the classical $P S N R$ as shown in Eq. 4.

$$
w P S N R=10 \log _{10}\left[\frac{\max (C)^{2}}{\|N V F(S-C)\|^{2}}\right](d B)
$$

where $N V F$ is defined as:

$$
N V F_{(i, j)}=\frac{1}{1+\sigma_{L_{(i, j)}}^{2}}
$$

where $\sigma_{L_{i, j}}^{2}$ denotes the local variance of an image in a window centered on the pixel with coordinates $(i, j)$.

The length of the secret data is another measurement criterion, which is defined as $E=\frac{K}{W H}(b p p)$, where $K$ is the number of the data message bits.

Figures 4(a) and 4(d) show one of the cover images used in the experiment and its histogram. Figures $4(\mathrm{~b})$ and $4(\mathrm{c})$ are the stego images resulting from the proposed method with $5 \%$ and $30 \%$ embedding rates. The visual differences between the cover and stego images cannot be discovered by the human eye, and 
Table 2: Image quality evaluation between the proposed method and Navas [7] technique with various embedding rates over 100 stego images.

The red values indicate the best result.

\begin{tabular}{c|c|cccc}
\hline Embed Rate & Method & MSE & PSNR & wPSNR & Avg. Difference \\
\hline \multirow{2}{*}{$5 \%$} & Navas [7] & 1.247 & 47.54 & 58.89 & 0.1188 \\
\cline { 2 - 6 } & Proposed & $\mathbf{0 . 0 9 6}$ & $\mathbf{5 8 . 4 6}$ & $\mathbf{6 0 . 8 4}$ & $\mathbf{0 . 0 6 0 8}$ \\
\hline \hline \multirow{2}{*}{$\mathbf{1 0 \%}$} & Navas [7] & 1.774 & 45.64 & 56.75 & 0.2527 \\
\cline { 2 - 6 } & Proposed & $\mathbf{0 . 2 2 5}$ & $\mathbf{5 4 . 6 8}$ & $\mathbf{5 7 . 0 4}$ & $\mathbf{0 . 1 3 2 1}$ \\
\hline \hline \multirow{2}{*}{$\mathbf{2 5 \%}$} & Navas [7] & - & - & - & - \\
\cline { 2 - 6 } & Proposed & $\mathbf{0 . 5 6 7}$ & $\mathbf{5 0 . 6 0}$ & $\mathbf{5 2 . 9 6}$ & $\mathbf{0 . 3 0 3 2}$ \\
\hline \hline \multirow{2}{*}{$\mathbf{4 0 \%}$} & Navas [7] & - & - & - & - \\
\cline { 2 - 6 } & Proposed & $\mathbf{0 . 8 0 1}$ & $\mathbf{4 9 . 1 0}$ & $\mathbf{5 1 . 5 5}$ & $\mathbf{0 . 3 7 7 9}$ \\
\hline \hline
\end{tabular}

even the histograms of the stego images (illustrated in Figures 4(e) and 4(f) are quite similar to that of the cover image.

In order to examine the performance of the presented scheme over other medical information hiding method, we compared our method with Navas's EPR hiding method [7] in term of visual quality and embedding rates. The obtained results that are listed in Table 2 indicate that the proposed method achieved better image quality for all metric measurements compared to Navas's method. Also, the proposed method provides a high embedding rate of up to $40 \%$, while Navas's method could only achieve an embedding rate of up to $13 \%$. The PSNR and wPSNR values of the proposed method are between (49.10 - 58.46) dB and (51.55 - 60.84) dB respectively. On the other hand, we have prevented any modifications to the ROI whereas Navas's method cannot guarantee this protection due to identifying the ROI from the low sub-band instead of identifying it in the spatial domain.

\section{Conclusion}

In this paper, a new and efficient medical image steganography is presented to hide the EPR data into the integer wavelet coefficients of RONI. It incorporates edge detection technique using overlapping blocks to identify and hide the secret data in the sharp regions of the image. The motivation behind using overlapping blocks is to improve the embedding payload by reducing the number of unused pixels. The difference between the cover and stego images is reduced by applying an XOR operation, which changes one bit at most to embed two secret bits into three pixel bits. The experimental results demonstrate improvements in the embedding capacity, and imperceptibility compared to one of the existing EPR hiding methods.

\section{References}

1. Singh Brar, A., Kaur, M.: High capacity, reversible data hiding using cdcs along with medical image authentication. International Journal of Signal Processing, 


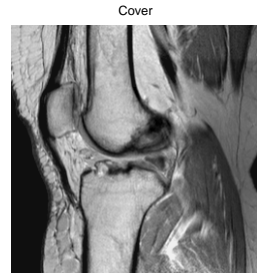

(a)

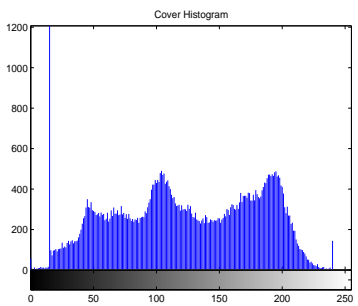

(d)

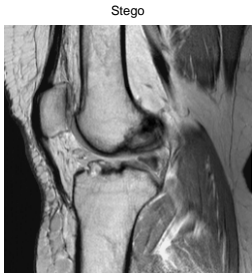

(b)

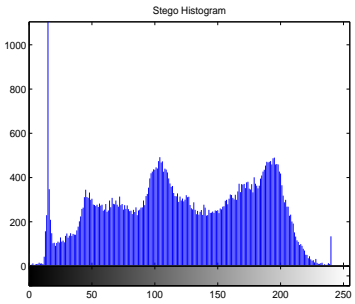

(e)

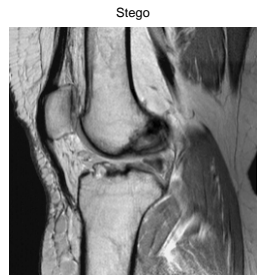

(c)

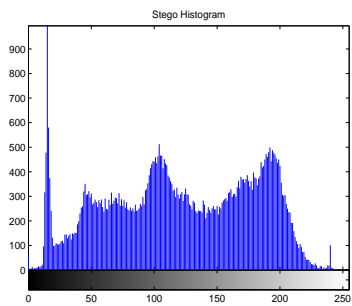

(f)

Fig. 4: (a) Cover Image $256 \times 256$, (b - c) Stego Images with $5 \%$ and $30 \%$ embedding rate, (d) Cover Image histogramand, and (e - f) Histograms of the corresponding stego images.

Image Processing \& Pattern Recognition 8 (2015)

2. Cheddad, A., Condell, J., Curran, K., Mc Kevitt, P.: Digital image steganography: Survey and analysis of current methods. Signal Processing 90 (2010) 727-752

3. Ramaiya, M.K., Hemrajani, N., Saxena, A.K.: Security improvisation in image steganography using des. In: Advance Computing Conference (IACC), 2013 IEEE 3rd International, IEEE (2013) 1094-1099

4. Al-Dmour, H., Al-Ani, A., Nguyen, H.: An efficient steganography method for hiding patient confidential information. In: Engineering in Medicine and Biology Society (EMBC), 2014 36th Annual International Conference of the IEEE, IEEE (2014) 222-225

5. Hamid, N., Yahya, A., Ahmad, R.B., Al-Qershi, O.M.: Image steganography techniques: an overview. International Journal of Computer Science and Security (IJCSS) 6 (2012) 168-187

6. Dumitrescu, S., Wu, X., Wang, Z.: Detection of lsb steganography via sample pair analysis. Signal Processing, IEEE Transactions on 51 (2003) 1995-2007

7. Navas, K., Thampy, S.A., Sasikumar, M.: Epr hiding in medical images for telemedicine. International Journal of Biomedical Sciences 3 (2008)

8. Zou, D., Shi, Y.Q., Ni, Z., Su, W.: A semi-fragile lossless digital watermarking scheme based on integer wavelet transform. Circuits and Systems for Video Technology, IEEE Transactions on 16 (2006) 1294-1300

9. Prabakaran, G., Bhavani, R., Rajeswari, P.: Multi secure and robustness for medical image based steganography scheme. In: Circuits, Power and Computing Technologies (ICCPCT), 2013 International Conference on, IEEE (2013) 1188-1193

10. Solanki, N., Malik, S.K., Chhikara, S.: Roni medical image watermarking using dwt and rsa. International Journal of Computer Applications 96 (2014) 